\title{
Non-serious case reports
}

The table below provides a list of the drugs associated with non-serious adverse events identified in the scientific literature over the last week.

\begin{tabular}{|c|c|c|}
\hline \multicolumn{3}{|l|}{ Non-serious case reports } \\
\hline Drug name(s) & Adverse event(s) & No. of cases \\
\hline Acitretin & Dry skin & 1 \\
\hline Alcohol & Injection site pain & 1 \\
\hline $\begin{array}{l}\text { Ananas comosus, Aspirin, Bisoprolol, Blueberry, } \\
\text { Clopidogrel, Docosahexaenoic acid, Radix } \\
\text { Glycyrrhizae, Telmisartan, Zingiber officiales }\end{array}$ & $\begin{array}{c}\text { Fatigue, Haemorrhage, Hypertension, Skin } \\
\text { injuries }\end{array}$ & 1 \\
\hline $\begin{array}{l}\text { Antithymocyte globulin, Cyclophosphamide, } \\
\text { Fludarabine }\end{array}$ & Gastrointestinal disorders & 1 \\
\hline Baclofen & Hypotension & 1 \\
\hline Ceritinib, Crizotinib, Lorlatinib & $\begin{array}{l}\text { Elevated aminotransferase levels, Nausea, } \\
\text { Oedema, Vomiting }\end{array}$ & 1 \\
\hline Ciclosporin & Eye Disorders, Ocular hyperaemia & 1 \\
\hline $\begin{array}{l}\text { Cinacalcet, Colecalciferol, Parenteral nutrition } \\
\text { supplements }\end{array}$ & Anorexia, Nausea, Pregnancy, Vomiting & 4 \\
\hline COVID 19 Vaccine Pfizer BioNTech & Exanthema & 1 \\
\hline Dabrafenib, Trametinib & Exanthema & 1 \\
\hline Dapagliflozin & Headache & 1 \\
\hline Dexamethasone, Methylprednisolone & Abnormal laboratory parameters & 1 \\
\hline Dexamethasone, Pembrolizumab, Pemetrexed & Dermatitis, Skin disorders & 1 \\
\hline Diclofenac, Tramadol & $\begin{array}{c}\text { Constipation, Diarrhoea, Dyspepsia, Flatulence, } \\
\text { Gastric disorders, Gastrointestinal disorders, } \\
\text { Nausea }\end{array}$ & 7 \\
\hline Doxycycline & Tremor & 1 \\
\hline Elexacaftor/Ivacaftor/tezacaftor & Urticaria & 1 \\
\hline Ferric carboxymaltose & Extravasation, Hyperpigmentation, Oedema & 1 \\
\hline Fluorouracil & Alopecia & 1 \\
\hline Gemcitabine & Nausea, Vomiting & 1 \\
\hline Hydroxycarbamide, Methotrexate, Vinblastine & Fatigue, Nausea & 1 \\
\hline Ibuprofen & Drug hypersensitivity & 1 \\
\hline Idelalisib, Venetoclax & Gastrointestinal disorders & 1 \\
\hline Immune globulin & Exanthema & 1 \\
\hline Ingenol mebutate & Inflammation & 6 \\
\hline Lenalidomide & Skin disorders & 1 \\
\hline Lenalidomide, Zanubrutinib & Exanthema & 1 \\
\hline Lisinopril & Cough & 1 \\
\hline mRNA 1273 & Chronic urticaria & 2 \\
\hline mRNA 1273, Tozinameran & Delayed hypersensitivity & 8 \\
\hline Mycophenolate mofetil, Tacrolimus & Exanthema, Skin disorders & 1 \\
\hline Piperacillin/tazobactam & Exanthema & 1 \\
\hline Prednisolone & Preeclampsia & 1 \\
\hline Propofol & Abnormal laboratory parameters & 1 \\
\hline SARS COV 2 vaccine inactivated Sinovac Biotech & Chilblains & 2 \\
\hline Tamsulosin & Hyperglycaemia & 1 \\
\hline Tigecycline & & 3 \\
\hline Tigecycline & Tooth disorders & 2 \\
\hline Tramadol/paracetamol & Nausea & 1 \\
\hline Trametinib & Elevated liver enzymes, Skin disorders & 1 \\
\hline
\end{tabular}

